1

\title{
Rich Club and reward network connectivity as endophenotypes for alcohol dependence: a DTI study
}

Nabi Zorlu ${ }^{1, *}$, Necip Çapraz ${ }^{2}$, Esra Oztekin ${ }^{1}$, Bas ak Bagci ${ }^{1}$, Maria A. Di Biase ${ }^{3}$, Andrew Zalesky $^{3}$, Fazıl Gelal ${ }^{4}$, Emre Bora ${ }^{3,5}$, Ercan Durmaz ${ }^{1}$, Lütfullah Bes $\square$ irog $\square$ lu$^{1}$, Aybala Sarıçiçek $^{1}$

${ }^{1}$ Department of Psychiatry, Katip Celebi University, Ataturk Training and Research Hospital, Izmir, Turkey

2

Department of Psychiatry, Cizre State Hospital, Sirnak, Turkey

3

Melbourne Neuropsychiatry Centre, Department of Psychiatry, The University of

Melbourne

and Melboume Health, Carton Sou

4

Department of Radiodiagnostics, Katip Celebi University, Ataturk Training and Research Hospital, Izmir, Turkey

${ }^{5}$ Department of Psychiatry, Dokuz Eylül University Medical School, Izmir, Turkey.

* Corresponding author at: Katip Celebi University Ataturk Training and Research Hospital, Department of Psychiatry, I $\square$ zmir, Turkey. Tel.: +90 232 2444444x1581.

E-mail address: zorlunabi@hotmail.com (N. Zorlu).

This is the author manuscript accepted for publication and has undergone full peer review but has not been through the copyediting, typesetting, pagination and proofreading process, which may lead to differences between this version and the Version of Record. Please cite this article as doi: $10.1111 / \mathrm{adb} .12599$

This article is protected by copyright. All rights reserved. 


\begin{abstract}
We aimed to examine the whole-brain white matter connectivity and local topology of reward system nodes in patients with alcohol use disorder (AUD) and unaffected siblings, relative to healthy comparison individuals. Diffusion-weighted MRI scans were acquired from 18 patients with AUD, 15 unaffected siblings of AUD patients and 15 healthy controls. Structural networks were examined using network-based statistic (NBS) and connectomic analysis. Connectomic analysis showed a significant ordered difference in normalized rich club organization (AUD < Siblings < Controls). We also found rank ordered differences (Control > Sibling > AUD) for both nodal clustering coefficient and nodal local efficiency in reward system nodes, particularly left caudate, right putamen and left hippocampus. NBS analyses showed that AUD group had significantly weaker connectivity than controls in the right hemisphere, mostly in the edges connecting putamen and hippocampus with other brain regions. Our results suggest that reward system network abnormalities, especially in subcortical structures, and impairments in rich-club organization might be related to the familial predisposition for AUD.
\end{abstract}

Key words: Alcohol use disorder, endophenotype, rich club, structural connectivity, 
white matter.

\section{INTRODUCTION}

Alcohol use disorder (AUD) is one of the most common mental disorders (Grant et al. 2015) and associated with psychosocial dysfunction (Kendler et al. 2017), disability (Grant et al. 2015), elevated mortality (Kendler et al. 2016) and physical and psychiatric comorbidities (Grant et al. 2015; Rehm 2011). Nevertheless, despite the increasing prevalence and disease burden of AUD, the neurobiological mechanisms underlying the disorder remain unclear.

Diffusion-weighted magnetic resonance imaging (MRI) can be used to investigate white matter (WM) in vivo. Fractional anisotropy (FA) is a measure of the degree to which water diffusion is constrained in the brain and is widely used as a general index of WM integrity (Kubicki et al. 2002). Damage to WM or demyelination along neuronal axons results in more isotropic water movement and manifests in lower FA values. Previous diffusion studies have reported decreased FA in both recently detoxified and in longterm abstinent AUD patients, most consistently in the corpus callosum, centrum 
semiovale, internal and external capsules, fornix, superior cingulate, and longitudinal fasciculi (Zahr \& Pfefferbaum 2017; Zorlu et al. 2013, 2014).

First-degree relatives of patients with AUD are at greater risk of developing the disorder and heritability of AUD is estimated at $50-85 \%$ (Long et al. 2017; Verhulst, Neale \& Kendler 2016), suggesting a large familial component to the disorder. Therefore, the investigation of unaffected first-degree relatives, in addition to AUD patients and healthy controls, may help to distinguish biomarkers of genetic risk without the confounding effects of the burden of illness, medication or clinical state. Previous studies examining WM integrity in genetically high-risk groups compared to controls found lower FA in frontal and parietal regions (Acheson et al. 2015; Herting, Fair \& Nagel 2011), no difference (Wetherill et al. 2012) or higher FA values in several regions throughout the brain (Squeglia et al. 2014). However, most of these studies were conducted in adolescents or young adults with a family history of alcoholism, and therefore, who were at risk of developing AUD in the future. Furthermore, previous studies (Acheson et al. 2015; Herting, Fair \& Nagel 2011; Wetherill et al. 2012; Squeglia et al. 2014) did not include a patient group in the analysis, which limits endophenotypic inference regarding WM abnormalities. Additionally, the degree of penetrance for candidate genes and environmental factors might also affect structural changes associated with genetic risk. Therefore, siblings of individuals with AUD provide a unique opportunity to investigate shared genetic and environmental risk factors of the disorder. 
Although previous diffusion studies have improved our understanding of WM alterations in AUD, they have typically applied a regional perspective, which limits interpretations at a whole-brain or network level. To overcome this limitation, recent studies have applied connectomics to examine alterations in the brain network topology of patients with psychiatric disorders (Lord et al. 2017). In connectomic analyses, a structural network of the brain is mapped that comprises cortical and subcortical regions (nodes) and WM connections between nodes (edges). Small-worldness is a key feature of brain network topology, which reflects an optimal balance between local segregation and global integration (Bullmore \& Sporns 2012). The most commonly used measures to characterize network topology are clustering coefficient (CC) and modularity (Q) for network segregation and characteristic path length (CPL) and global efficiency (GE) for network integration. Briefly, higher levels of $\mathrm{CC}$ and $\mathrm{Q}$ suggest the presence of locally connected clusters or modules implicated in specialized information processing. In addition, low CPL and high GE levels suggest a more globally integrated network. Another important feature of the brain network topology is rich-club organization, which is thought to represent a backbone facilitating network integration (van den Heuvel \& Sporns 2013). In short, some brain regions are connected to a large number of other regions. Moreover, some of these so-called hub regions are also more likely to be interconnected among each other than expected by chance. This densely interconnected group of hub regions is called a rich club. Importantly, previous studies 
reported genetic influences on structural network topology (Bohlken et al. 2014; Fulcher \& Fornito 2015). Furthermore, two previous studies reported intermediate levels of richclub organization, clustering and efficiency between patients and controls in siblings of patients with schizophrenia (Collin et al. 2014; Lo et al. 2015). Therefore, given the heritability of AUD, we hypothesize that the same patterns are evident in the siblings of patients with AUD.

The first connectomic analysis of AUD did not identify any abnormalities in AUD individuals in both global functional network topology and local topology of the striatal nodes, using functional MRI (Sjoerds et al. 2017). In contrast, the second study found higher $\mathrm{CC}$, indicating higher clustered and segregated network topology and information processing in a functional network of AUD patients compared to controls (Morris et al. 2017). A third study was conducted in relatives of patients with AUD on functional networks (Holla et al. 2017), which found reduced CC, small-worldness, and local network efficiency in substance-naive high-risk male offspring with a family history of AUD compared to controls without a family history of AUD. However, it is not known whether abnormalities in functional networks in AUD reflect underlying changes in structural topology. Given the evidence for WM abnormalities in AUD, connectomic analysis using structural networks might provide further insight about the mechanisms underlying AUD. 
Reward processing abnormalities are one of the major models for addiction (Volkow \& Morales 2015) and thought to be mediated by cortico-limbic-striatal brain networks, particularly medial orbitofrontal cortex (mOFC) (Peters \& D'Esposito 2016; Zald et al. 2014), striatum (Luijten et al. 2017; Richter et al. 2017), hippocampus (Richter et al. 2017) and amygdala (Sescousse et al. 2013). Furthermore, reward processing abnormalities seem to be an important risk for developing AUD (Hill \& Brien 2015). However, whether abnormalities in nodes of the reward system are premorbid conditions or consequences of alcohol use remains unclear. Given the heritability of AUD and brain network topology (Bohlken et al. 2014; Fulcher \& Fornito 2015), examining reward network topology in unaffected siblings of patients with AUD might provide insight into whether reward system abnormalities might be a candidate endophenotype.

Here, we applied the network-based statistic (NBS) (Zalesky, Fornito \& Bullmore 2010) to identify white matter connections that were significantly altered in AUD individuals and unaffected siblings, relative to healthy comparison individuals. We also compared global and local graph measures between these groups to determine whether white matter connectivity alterations are related to familial liability. We hypothesized that siblings have intermediate levels of rich-club organization and local topology of reward system nodes between patients with AUD and controls. 


\section{MATERIALS AND METHODS}

\section{Participants}

A total of 18 patients with AUD who had been abstinent for at least 2 weeks before scanning, 15 siblings of AUD patients and 15 healthy controls were enrolled in the study. All subjects were male and right-handed. The groups were matched for age and years of education. The advantage of studying subjects abusing various substances in Turkey is that there are groups of subjects with single substance abuse, particularly alcohol. Hence, we recruited patients with AUD who abused only alcohol to date.

Exclusion criteria for the alcohol dependence group were as follows: (1) any lifetime substance use other than alcohol (except nicotine), (2) current or past history of any serious psychiatric illness, including any psychotic disorder, bipolar disorder, (3) current use of psychotropic medication (4) current or past history of any significant neurological disorders, (5) history of loss of consciousness for more than $30 \mathrm{~min},(6)$ any severe hepatic, endocrine, renal disease, and (7) any contraindications for MRI scanning (metal implants, pacemakers, etc.). Siblings and control subjects met the same criteria as patients, except for the history of alcohol dependence. All subjects were interviewed using the Structured Clinical Interview for DSM-IV Axis I Disorders (First et al. 1999) to exclude participants with past or current comorbid Axis I diagnoses and to confirm the diagnosis of alcohol dependence in the clinical group. 
AUD patients were interviewed in order to determine the age at which they started drinking, the length of time they had alcohol dependence and duration from the last alcohol consumption. Monitoring of blood and urine for the presence of alcohol, amphetamines, barbiturates, benzodiazepines, cocaine, cannabis, and opiates was performed to assure sobriety at the day of scanning for only patients with AUD.

All subjects gave written informed consent to participate in the study. The study was approved by local research and ethics committees.

\section{Imaging protocol}

MRI was performed using a 1.5T MR system (GE SignaHDxt, General Electric Medical Systems, Milwaukee, WI, USA). Diffusion imaging data were acquired in 41 diffusion gradient directions (b-value $=1000 \mathrm{~s} / \mathrm{mm}^{2}$ ) and a single non-diffusion weighted reference image using a sequence optimized to collect diffusion-weighted images (repetition time $=6500 \mathrm{~ms}$, echo time $=90 \mathrm{~ms}$, voxel size $=1 \times 1 \times 2 \mathrm{~mm}^{3}$ ).

\section{Data pre-processing and network construction}

DTI data were analyzed using FMRIB's (Oxford Centre for Functional MRI of the Brain) Diffusion Toolbox, which is part of FSL (FMRIB Software Library) (Smith et al. 2004). 
Motion and eddy current artifacts were corrected using FSL's "eddy-correct" command. A brain mask of the non-diffusion-weighted image was created using FSL's Brain Extraction Tool (Smith 2002). Diffusion tensors were then calculated with FSL DTIFIT for whole brain volumes, and the resulting FA volume of each subject was then registered to the FMRIB58_FA template using the FSL nonlinear registration tool FNIRT to obtain the warp field from native to standard space. The nonlinear warp was initialized with an affine registration generated with the FSL linear registration tool FLIRT.

Deterministic whole-brain streamline counts were generated using the fiber assignment by continuous tracking (FACT) algorithm using the TrackVis Diffusion Toolkit (trackvis.org) in native diffusion space for each individual after rotation corrections were applied (Leemans \& Jones 2009); parameters included a $35^{\circ}$ degree angle threshold, twenty seeds were placed in every voxel and a minimum streamline length of $20 \mathrm{~mm}$ was used.

Network nodes were based on the 116 cortical, subcortical, and cerebellar regions comprising the automated anatomical labeling (AAL) atlas (Tzourio-Mazoyer et al. 2002). AAL labels were mapped to each individual's native space using the inverse of the warp field previously computed. Each individual's network was represented with a symmetric $\mathrm{N} \times \mathrm{N}$ connectivity matrix, where $\mathrm{N}$ denoted the number of nodes. Each element of the connectivity matrix was populated with the number of streamlines 
between the corresponding pair of regions (streamline count), which served as a measure of inter-regional connection strength.

\section{NBS analyses}

AUD patients and siblings were separately compared with controls. For each of the comparisons (AUD vs Controls and Siblings vs Controls), all connectivity matrices were subject to a group threshold, applied across all subjects, to eliminate spurious connections (de Reus \& van den Heuvel 2013). This involved eliminating any pair of regions that was not interconnected by one or more streamlines in at least $25 \%$ of all individuals. In performing the NBS calculations, to localize differences in the connection strengths (streamline count) to specific networks, while controlling the family-wise error (FWE), the primary threshold for each inter-regional connection was set to a $\mathrm{t}$ value $=$ 2.3 (10000 permutations).

\section{Graph theoretical analyses}

For each dataset, a threshold was applied to each weighted undirected structural connectivity matrix (edges with less than 3 streamlines were set to zero) to reduce false positive connections and graph theory metrics were computed with the brain connectivity toolbox (Rubinov \& Sporns 2010). 
First, we examined global network measures including clustering coefficient (CC), modularity $(\mathrm{Q})$, characteristic path length $(\mathrm{CPL})$, global efficiency $(\mathrm{GE})$, and smallworldness. CC is defined as the fraction of triangles around a node and is equivalent to the fraction of node's neighbors that are neighbors of each other (Rubinov \& Sporns 2010). Q quantifies the degree to which the network may be subdivided into such clearly delineated groups (Rubinov \& Sporns 2010). CPL is the average shortest path length in the network and the GE is the average inverse shortest path length in the network (Rubinov \& Sporns 2010). CC and CPL were normalized by 1,000 randomized null network preserving the number of nodes, edges, and degree distribution (CCnorm and CPLnorm). Small-worldness was calculated as; CCnorm/CPLnorm. Additionally, we also examined the nodal network measures in the a priori selected ROls, including bilateral putamen and caudate nucleus, hippocampus, amygdala and medial OFC, based on previous research as mentioned above, including the nodal local efficiency (Enodal) and clustering coefficient (CCnodal). Enodal is the GE computed on the neighborhood of the node (Rubinov \& Sporns 2010).

Second, we calculated the rich club coefficient as the connection density of the subnetwork defined by the set of nodes with degrees exceeding $k$. The degree threshold, $k$, was systematically varied to test rich club organization at different levels. The rich club coefficient was normalized (RCnorm) with respect to an ensemble of 1000 surrogate networks with the same number of nodes, edges, and degree distribution as 
the empirical data, but which was randomized in all other respects. RCnorm $>1$ over a range of $\mathrm{k}$ implies the presence of a rich-club in a network (van den Heuvel \& Sporns 2011).

Nonparametric Jonckheere-Terpstra (JT) permutation analysis (10000 permutations) was applied to test whether graph metrics have a familial pattern (AUD > Siblings > Controls or Controls > Siblings > AUD). False discovery rate (FDR) correction for multiple comparisons (Benjamini \& Hochberg 1995) was applied to the global (5 statistical tests), nodal (10 statistical tests, one per region) and rich-club organization (16 statistical tests, one per k levels) analyses. We used additional post-hoc independent sample t test to compere graph metrics between patients with AUD versus controls and siblings versus controls if $\mathrm{JT}$ test indicated significant differences after FDR correction.

\section{Robustness of main results}

The stability of results for the global, nodal and rich-club organization was checked using a variety of thresholds on the streamline count. We utilized five additional thresholds of streamline counts $\geq 0,1,2,4$ and 5 . We also normalized edge values to remove the possible influence of the streamline counts connecting pairs of regions across individuals.

This article is protected by copyright. All rights reserved. 
To check the stability of NBS results, we applied different thresholds across a range from $5 \%$ to $\% 30$ in steps of $5 \%$.

\section{RESULTS}

\section{Demographic and alcohol use variables}

Groups were comparable for age, educational level and smoking status. Table 1 shows the demographic and alcohol use variables for the 3 groups.

\section{Table 1 here}

\section{Global Metrics}

JT testing did not show ordered differences for CCnorm, CPLnorm, small-worldness and GE. We found ordered difference for Q, such that Patients > Siblings > Controls at an uncorrected level but not after FDR correction (Table 2).

\section{Table 2 here}

\section{Reward System Node-Specific Metrics}

There were significant ordered differences for both CCnodal and Enodal (Control > Sibling > AUD) in the left hippocampus and caudate and right putamen that survived 
FDR correction. Post-hoc $\mathrm{t}$ tests demonstrated significantly reduced CCnodal and Enodal in AUD compared with controls in all nodes. Siblings showed a trend for both CCnodal $(p=0.70)$ and Enodal $(p=0.054)$ in right putamen (Figure 1) (see Supporting Information Table S1 and Table S2 for further information).

Figure 1 here

\section{Rich-Club Organization}

There was a significant ordered difference in normalized rich club organization after FDR correction (AUD < Siblings < Controls) over the range of $k$ from 13 to 17 (Figure 2). Post-hoc $\mathrm{t}$ tests demonstrated significantly reduced normalized rich club organization in AUD compared with controls in all significant $k$ values. Siblings showed significantly reduced $\mathrm{RCnorm}$ in $\mathrm{k}=17(\mathrm{p}=0.046)$ and a trend for $\mathrm{k}=16(\mathrm{p}=0.071)$.

\section{Figure 2 here}

\section{Structural Connectivity}

NBS analyses identified a single network of significantly decreased structural connectivity in AUD group compared to controls ( $t=2.3, p=0.014$; Figure 3 ). The network was on the right hemisphere and consisted of ten nodes connected by nine edges. These connections linked the putamen with the inferior frontal gyrus (both triangular and 
opercular parts), inferior temporal gyrus and supramarginalgyrus. Another three edges linked the hippocampus with inferior temporal gyrus, middle occipital gyrus and superior occipital gyrus. Two further edges linked the superior frontal gyrus with caudate and inferior frontal gyrus, opercular part (Figure 3). We further examined the nine edges showing decreased streamline count in AUD group to see whether streamline counts also show significant ordered differences similar to topological analyses. All edges except edge between the right putamen and inferior frontal gyrus triangular part showed significant ordered differences (AUD < Siblings < Controls) after FDR correction (see Supporting Information Table S3 for further information). There were no differences between AUD group and controls at higher supra-thresholds $(t=2.5, t=3.0)$.

There was a trend for a reduced structural connectivity in siblings compared to controls $(t=2.3, p=0.066)$. The network was on the left hemisphere and connections linked the middle temporal gyrus with inferior temporal gyrus and inferior occipital gyrus.

There were no edges showing increased structural connectivity in AUD group or siblings compared with controls with NBS.

\section{Figure 3 here}

This article is protected by copyright. All rights reserved. 
We did not find any significant correlations between the alcohol use variables and richclub organization, reward system metrics and streamline counts in the AUD group.

\section{Reproducibility of the findings}

Reward system node-specific metrics were generally robust to the choice of streamline threshold. In particular, CCnodal and Enodal in the left caudate and right putamen remained statistically significant for all streamline thresholds. Enodal in the left hippocampus was statistically significant for 5 of 7 thresholds and CCnodal was statistically significant for 4 of 7 thresholds (see Supporting Information Table S4 and S5 for further information). Global metrics remained unchanged across all streamline thresholds. On the other hand, rich-club organization was only significant for streamline count threshold of $\geq 1(k=19, p=0.032 ; k=20, p=0.046 ; k=21, p=0.046)$.

All nine edges that we found significantly decreased in AUD group compared to controls remained stable for the various thresholds $(5 \%, p=0.006 ; 10 \%, p=0.003 ; 15 \%, p=$ $0.012 ; 20 \%, p=0.014)$. Only for the $30 \%(p=0.017)$, edge between putamen with the supramarginalgyrus did not remain significant.

\section{DISCUSSION}

This article is protected by copyright. All rights reserved. 
We examined AUD patients, unaffected siblings of AUD patients and healthy controls in order to identify alterations in anatomical connectivity that may be associated with the familial risk for AUD. We found rank ordered differences in network topology in reward system nodes (Control>Siblings>AUD), particularly caudate, putamen and hippocampus and abnormal rich club organization in AUD patients and unaffected siblings of AUD patients. Our results suggest that rich club organization and subcortical connectivity abnormalities might be imaging endophenotypes.

We found significant rank ordered differences of CCnodal and Enodal, such that values were lowest in AUD group, intermediate in sibling group and highest in controls, in the left caudate and right putamen and left hippocampus. The lower levels of CCnodal observed in these nodes reflect reduced connectivity between the neighbors of these nodes that suggest weaker specialized information processing and lower levels of Enodal suggest less efficient (with more edges) connection of these nodes with all other nodes in the network. Striatum as well as hippocampus has been implicated in rewardrelated and motivated behaviors which are thought to be a core mechanism of addiction (Volkow \& Morales 2015). A recent PET study found increased reward-induced dopamine release in the bilateral caudate nucleus, putamen and ventral striatum and extensive reward-induced dopamine release in dorsal striatum was associated with better reinforcement learning task performance in the healthy volunteers (Kasanova et al. 2017). Furthermore, another recent study found that genetic variations associated 
with dopamine D2 receptor expression affect modulation of striatal and hippocampal brain responses during encoding of reward-predicting items and healthy controls with lower dopamine D2 receptor expression exhibited better reward-related memory (Richter et al. 2017). Striatal and hippocampal abnormalities were also found in the relatives of substance dependent subjects with different neuroimaging methods. A PET study in younger drinkers with short drinking histories found an approximately fourfold striatal dopamine increase after tasting a preferred alcoholic beverage in subjects with a first-degree relative with AUD compared to family history-negative subjects (Oberlin et al. 2013). Additionally, a voxel-based morphometry study found larger medial temporal lobe and putamen volumes in the siblings of stimulant dependent patients (Ersche et al. 2012). Thus, it is possible that reward system alterations in subcortical structures reflect a genetic predisposition rather than a consequence of alcohol dependence. Speculatively, these abnormalities might lead to increased reward sensitivity, which may confer greater risk for initiation and maintenance of alcohol use.

We also found impaired rich-club organization in patients with AUD and to a lesser degree, in siblings of AUD patients compared to controls (AUD $<$ Sibling $<$ Controls) that suggest reduced structural connectivity among the central hubs in the AUD and sibling groups. These findings are in line with previous studies reporting heritability of structural brain connectivity features (Bohlken et al. 2014; Fulcher and Fornito 2015). Rich-club organization is thought to mediate the integration of information between segregated 
parts of the brain network (van den Heuvel \& Sporns 2013). However, the clinical importance of rich-club organization is still an open question. A recent study reported stronger rich club organization is associated with better cognitive performance in healthy volunteers (Baggio et al. 2015). Therefore, our results may partly explain cognitive abnormalities reported in both alcoholics (Le Berre, Fama \& Sullivan 2017) and firstdegree relatives of patients with AUD (Cservenka 2016).

NBS analyses showed that AUD group had significantly weaker connectivity than controls in the right hemisphere, mostly in the edges connecting putamen and hippocampus with other brain regions. There were no differences between sibling and control groups but sibling group showed intermediate streamline numbers in the edges that was found lower in AUD group compared to controls. We found four decreased edges included in putamen. Animal studies have shown that dorsolateral striatum (putamen in humans) is essential for habitual alcohol seeking (Corbit \& Janak 2016) and according to contemporary theories of addiction, putamen is one of the core regions for the transition from goal-directed reward related behavior into habitual consumption of alcohol (Everitt \& Robbins 2016). However, habitual alcohol seeking is not enough to explain addiction process and compulsive alcohol seeking despite negative consequences is essential for addiction. Compulsive drug seeking is thought to be due to disrupted cortical control, especially prefrontal cortex, over behavior with the longterm drug use (Everitt \& Robbins 2016). Considering that the right inferior frontal gyrus 
known to mediate response inhibition (Fuentes-Claramonte et al. 2016), our finding of reduced connectivity between right putamen and right inferior frontal gyrus could be interpreted as evidence of reduced fronto-striatal control due to the long-term alcohol use. However, due to cross-sectional nature of our study, we are unable to establish a clear temporal relationship between alcohol use and reduced connectivity between cortical and subcortical regions.

It is also important to note that middle aged sibling group in our study had not developed AUD despite the fact that they shared reward system alterations with AUD group. This might suggest that more risk factors are involved in the development of AUD and/or that protective factors might also prevent siblings from developing AUD. For example siblings showed intermediate values between AUD group and controls while siblings were not significantly different from controls on these values except rich-club organization. Lower number or expression of high-risk genes in siblings compared to patients with AUD might be a possible explanation for intermediate levels that was shown in our study. Another possible explanation might be the environmental factors differentiated between siblings and patients might contribute to AUD in patients but not in siblings on the basis of distributed structural network topology in both groups. A longitudinal study would be required to elucidate these relationships.

This article is protected by copyright. All rights reserved. 
The current study has several potential limitations. The most obvious is the crosssectional nature of the study, so that potential differential changes in WM measurements over the course of illness in AUD remain to be directly established. Second, our sample was relatively small, which might reduce the power of the group comparison especially for the unaffected sibling group as not all individuals in this group are likely to be carrying the susceptibility gene to AUD. Third, the field strength of 1.5 Tesla should be noted. Fourth, DTI-based deterministic tractography has been shown to have a limited capacity for resolving crossing, converging or diverging fibers which might lead to unreliable connectivity matrices that comprise false positive connections (Jbabdi \& Johansen-Berg 2011, Zalesky et al. 2016, Maier-Hein et al. 2017). Fifth, due to an absence of standardized methodological criteria, the choice of streamline thresholds is rather arbitrary, although the majority of our findings were robust to the choice of threshold. Rich-club organization was most sensitive to the choice of threshold and thus these findings should be interpreted cautiously with respect to the contribution of weak and potentially spurious connections. Finally, we cannot extend our findings to females with AUD and their siblings as our sample included only men.

In conclusion, our results suggest that reward system network abnormalities, especially in subcortical structures, and impairments in rich-club organization might be related to the familial predisposition for AUD.

This article is protected by copyright. All rights reserved. 


\title{
Acknowledgements
}

Andrew Zalesky was supported by NHMRC Career Development Fellowship (GNT1047648). Maria Di Biase was supported by the Rotary Health lan Scott PhD Scholarship in Mental Health.

\section{Declarations of interest}

All authors declare that they have no conflicts of interest.

\section{AUTHORS CONTRIBUTION}

\begin{abstract}
NZ, NC, AS, and LB were responsible for the study concept and design. NC, EO, BB, ED, and FG contributed to the acquisition of imaging data. NZ analyzed the data. NZ drafted the manuscript. AZ, EB, and MAD provided critical revision of the manuscript for important intellectual content. All authors critically reviewed content and approved final version for publication.
\end{abstract}

\section{References}

This article is protected by copyright. All rights reserved. 
Acheson A, Wijtenburg SA, Rowland LM, Winkler AM, Gaston F, Mathias CW, Fox PT, Lovallo WR, Wright SN, Hong LE, Dougherty DM (2014) Assessment of whole brain white matter integrity in youths and young adults with a family history of substance-use disorders. Hum Brain Mapp 35:5401-5413.

Baggio HC, Segura B, Junque C, de Reus MA, Sala-Llonch R, Van den Heuvel MP (2015) Rich club organization and cognitive performance in healthy older participants. J Cogn Neurosci 27:1801-1810.

Benjamini $Y$, Hochberg $Y$ (1995) Controlling the false discovery rate- a practical and powerful approach to multiple testing. J R Stat Soc Ser B-Methodol. 57:289-300.

Bohlken MM, Mandl RC, Brouwer RM, van den Heuvel MP, Hedman AM, Kahn RS, Pol H, Hilleke E (2014) Heritability of structural brain network topology: A DTI study of 156 twins. Hum Brain Mapp 35:5295-5305.

Bullmore E, Sporns O (2012) The economy of brain network organization. Nat Rev Neurosci 13:336-349.

Collin G, Kahn RS, de Reus MA, Cahn W, van den Heuvel MP (2014) Impaired Rich Club Connectivity in Unaffected Siblings of Schizophrenia Patients. Schizophr Bull 
40:438- 448.

Corbit LH, Janak PH (2016) Habitual alcohol seeking: Neural bases and possible relations to alcohol use disorders. Alcohol Clin Exp Res 40:1380-1389.

Cservenka A (2016) Neurobiological phenotypes associated with a family history of alcoholism. Drug Alcohol Depend 158:8-21.

de Reus MA, van den Heuvel MP (2013) Estimating false positives and negatives in brain networks. Neuroimage 70:402-409.

Ersche KD, Jones PS, Williams GB, Turton AJ, Robbins TW, Bullmore ET (2012) Abnormal brain structure implicated in stimulant drug addiction. Science 335:601-604.

Everitt BJ, Robbins TW (2016) Drug addiction: updating actions to habits to compulsions ten years on. Annu Rev Psychol 67:23-50.

First MB, Spitzer RL, Gibbon M, Williams JBW (1997) Structured clinical interview for DSM-IV axis I disorders (SCID-I), clinical version. American Psychiatric Press, Washington D.C. and London.

This article is protected by copyright. All rights reserved. 
Fuentes-Claramonte P, Avila C, Rodriguez-Pujadas A, Costumero V, Ventura-Campos N, Bustamante JC, Rosell-Negre P, Barrós-Loscertales A (2016) Inferior frontal cortex activity is modulated by reward sensitivity and performance variability. Biol Psychol $114: 127-137$.

Fulcher BD, Fornito A (2015) A transcriptional signature of hub connectivity in the mouse connectome. Proc Natl Acad Sci 113:1435-1440.

Grant BF, Goldstein RB, Saha TD, Chou SP, Jung J, Zhang H, Pickering RP, Ruan WJ, Smith SM, Huang B, Hasin DS (2015) Epidemiology of dsm-5 alcohol use disorder: Results from the national epidemiologic survey on alcohol and related conditions III. JAMA Psychiatry 72:757-766.

Herting MM, Fair D, Nagel BJ (2011) Altered fronto-cerebellar connectivity in alcoholnaive youth with a family history of alcoholism. Neuroimage 54:2582-2589.

Hill SY, O'Brien J (2015) Psychological and neurobiological precursors of alcohol use disorders in high risk youth. Curr Addict Rep 2:104-113.

This article is protected by copyright. All rights reserved. 
Holla B, Panda R, Venkatasubramanian G, Biswal B, Bharath RD, Benegal V (2017) Disrupted resting brain graph measures in individuals at high risk for alcoholism. Psychiatry Res 30:54-64.

Jbabdi S, Johansen-Berg H (2011) Tractography: Where do we go from here? Brain Connectivity 1: 169-183.

Kasanova Z, Ceccarini J, Frank MJ, Amelsvoort V, Booij J, Heinzel A, Mottaghy F, MyinGermeys I (2017) Striatal dopaminergic modulation of reinforcement learning predicts reward-oriented behavior in daily life. Biol Psychol 127:1-9.

Kendler KS, Ohlsson H, Karriker-Jaffe KJ, Sundquist J, Sundquist K (2017) Social and economic onsequences of alcohol use disorder: a longitudinal cohort and co-relative analysis. Psychol Med 47:925-935.

Kendler KS, Ohlsson H, Sundquist J, Sundquist K (2016) Alcohol use disorder and mortality across the lifespan: A longitudinal cohort and co-relative analysis. JAMA Psychiatry 73:575-581.

This article is protected by copyright. All rights reserved. 
Kubicki M, Westin CF, Maier SE, Mamata H, Frumin M, Ersner-Hershfield H, Kikinis R, Jolesz FA, McCarley R, Shenton ME (2002) Diffusion tensor imaging and its application to neuropsychiatric disorders. Harv Rev Psychiatry 10:324-336.

Le Berre AP, Fama R, Sullivan EV (2017) Executive functions, memory, and social cognitive deficits and recovery in chronic alcoholism: a critical review to inform future research. Alcohol Clin Exp Res Jun:15. doi: 10.1111/acer.13431. [Epub ahead of print]

Leemans A, Jones DK (2009) The B-matrix must be rotated when correcting for subject motion in DTI data. Magn Reson Med 61:1336-1349.

Lo CY, Su TW, Huang CC, Hung CC, Chen WL, Lan TH, Lin CP, Bullmore ET (2015) Randomization and resilience of brain functional networks as systems-level endophenotypes of schizophrenia. Proc Natl Acad Sci 112:9123-9128.

Lord LD, Stevner AB, Deco G, Kringelbach ML (2017) Understanding principles of integration and segregation using whole-brain computational connectomics $\square$ : implications for neuropsychiatric disorders. Philos Trans A Math Phys Eng Sci 375:20160283.

This article is protected by copyright. All rights reserved. 
Long EC, Lönn SL, Sundquist J, Sundquist K, Kendler KS (2017) A national swedish longitudinal twin-sibling study of alcohol use disorders among males. Addiction Mar:26. doi: 10.1111/add.13833. [Epub ahead of print]

Luijten M, Schellekens AF, Ku $\square$ hn S, Machielse MW, Sescousse G (2017) Disruption of reward processing in addiction: An image-based meta-analysis of functional magnetic resonance imaging studies. JAMA Psychiatry 74:387-398.

Maier-Hein KH, Neher PF, Houde JC, Côté MA, Garyfallidis E, Zhong J, ... Reddick WE (2017) The challenge of mapping the human connectome based on diffusion tractography. Nat Commun (8), 1349. doi: 10.1038/s41467-017-01285-x.

Morris LS, Baek K, Tait R, Elliott R, Ersche KD, Flechais R, McGonigle J, Murphy A, Nestor LJ, Orban C, Passetti F (2017) Naltrexone ameliorates functional network abnormalities in alcohol-dependent individuals. Addict Biol Feb:28. doi: 10.1111/adb.12503. [Epub ahead of print]

Oberlin BG, Dzemidzic M, Tran SM, Soeurt CM, Albrecht DS, Yoder KK, Kareken DA (2013) Beer flavor provokes striatal dopamine release in male drinkers mediation family history of alcoholism. Neuropsychopharmacology 38:1617-1624. 
Peters J, D'Esposito M (2016) Effects of medial orbitofrontal cortex lesions on selfcontrol in intertemporal choice. Curr Biol 26:2625-2628.

Rehm J (2011) The risks associated with alcohol use and alcoholism. Alcohol Res Health $34: 135-143$.

Richter A, Barman A, Wüstenberg T, Soch J, Schanze D, Deibele A, Behnisch G, Assmann A, Klein M, Zenker M, Seidenbecher C (2017) Behavioral and neural manifestations of reward memory in carriers of low-expressing versus high-expressing genetic variants of the dopamine D2 receptor. Front Psychol May:1. doi:10.3389/fpsyg.2017.00654.

Rubinov M, Sporns O (2010) Complex network measures of brain connectivity: Uses and interpretations. Neuroimage 52:1059-1069.

Sescousse G, Caldu X, Segura B, Dreher JC (2013) Processing of primary and secondary rewards: A quantitative meta-analysis and review of human functional neuroimaging studies. Neurosci Biobehav Rev 37:681-696.

Sjoerds Z, Stufflebeam SM, Veltman DJ, Van den Brink W, Penninx BW, Douw L (2017) Loss of brain graph network efficiency in alcohol dependence. Addict Biol 22:523-534. 
Smith SM, Jenkinson M, Woolrich MW, Beckmann CF, Behrens TE, Johansen-Berg H, Bannister PR, De Luca M, Drobnjak I, Flitney DE, Niazy RK (2004) Advances in functional and structural MR image analysis and implementation as FSL. Neuroimage 23(Suppl 1):208-219.

Smith SM (2002) Fast robust automated brain extraction. Hum Brain Mapp 17:143-155.

Squeglia LM, Jacobus J, Brumback T, Meloy MJ, Tapert TS (2014) White matter integrity in alcohol-naive youth with a family history of alcohol use disorders. Psychol Med 44:2775-2786.

Tzourio-Mazoyer N, Landeau B, Papathanassiou D, Crivello F, Etard O, Delcroix N, Mazoyer B, Joliot M (2002) Automated anatomical labeling of activations in SPM using a macroscopic anatomical parcellation of the MNI MRI single-subject brain. Neuroimage 15:273-289.

van den Heuvel MP, Sporns O (2013) Network hubs in the human brain. Trends Cogn Sci 17:683-696.

This article is protected by copyright. All rights reserved. 
van den Heuvel MP, Sporns O (2011) Rich-Club organization of the human connectome. J Neurosci 31:15775-15786.

Verhulst B, Neale MC, Kendler KS (2016) The heritability of alcohol use disorders: a meta-analysis of twin and adoption studies. Psychol Med 45:1061-1072.

Volkow ND, Morales M (2015) The brain on drugs: From reward to addiction. Cell 162:712-725.

Wetherill RR, Bava S, Thompson WK, Boucquey V, Pulido C, Yang TT, Tapert SF (2012) Frontoparietal connectivity in substance-naive youth with and without a family history of alcoholism. Brain Res 1432:66-73.

Zahr NM, Pfefferbaum A (2017) Alcohol's effects on the brain: Neuroimaging results in humans and animal models. Alcohol Research: Current Reviews 38:e1-24.

Zald DH, McHugo M, Ray KL, Glahn DC, Eickhoff SB, Laird AR (2014) Meta-analytic connectivity modeling reveals differential functional connectivity of the medial and lateral orbitofrontal. Cereb Cortex 24:232-248.

This article is protected by copyright. All rights reserved. 
Zalesky A, Fornito A, Bullmore ET (2010) Network-based statistic: Identifying differences in brain networks. Neuroimage 53:1197-1207.

Zalesky A, Fornito A, Cocchi L, Gollo LL, van den Heuvel MP, Breakspear M (2016) Connectome sensitivity or specificity: which is more important? Neurolmage 142:407420.

Zorlu N, Gelal F, Kuserli A, Cenik E, Durmaz E, Saricicek A, Gulseren S (2013) Abnormal white matter integrity and decision-making deficits in alcohol dependence. Psychiatry Res 214:382-388.

Zorlu N, Karavul Ucman T, Gelal F, Colak Kalayci C, Polat S, Saricicek A, Zorlu PK, Gulseren S (2014) Abnormal white matter integrity in long-term abstinent alcohol dependent patients. Psychiatry Res 224:42-48.

This article is protected by copyright. All rights reserved. 
Table 1 Demographics and clinical characteristics of in the patients with alcohol use disorder (AUD), siblings and controls.

AUD $(n=18) \quad$ Siblings $(n=15) \quad$ Controls $(n=15) \quad$ Statistics

\begin{tabular}{|c|c|c|c|c|}
\hline Age & $40.8 \pm 6.4$ & $42.5 \pm 8.1$ & $42.8 \pm 7.2$ & $\mathrm{~F}=0.391, \mathrm{p}=0.679$ \\
\hline Education (years) & $8.9 \pm 3.2$ & $8.6 \pm 3.1$ & $8.7 \pm 4.0$ & $\mathrm{~F}=0.240, \mathrm{p}=0.788$ \\
\hline Smokers & $14 / 18$ & $9 / 15$ & $10 / 15$ & $\chi=1.248, p=0.536$ \\
\hline $\begin{array}{l}\text { Duration of } \\
\text { dependence (years) }\end{array}$ & $12.0 \pm 6.5$ & & & \\
\hline $\begin{array}{l}\text { Duration of } \\
\text { abstinence (days) }\end{array}$ & $38.9 \pm 24.9$ & & & \\
\hline
\end{tabular}


Age at first use $\quad 17.6 \pm 5.2$

(years)

Data are presented as mean \pm standard deviation unless otherwise indicated.

Table 2 Global metrics of brain WM structural networks in the patients with alcohol use disorder (AUD), siblings and controls.

\begin{tabular}{lcccc}
\hline & AUD $(\mathrm{n}=18)$ & Siblings $(\mathrm{n}=15)$ & Controls $(\mathrm{n}=15)$ & P value \\
& & & & \\
\hline CCnorm & $4.713 \pm 0.593$ & $4.561 \pm 0.554$ & $4.564 \pm 0.494$ & 0.40 \\
CPLnorm & $2.515 \pm 1.333$ & $2.777 \pm 1.179$ & $2.419 \pm 0.466$ & 0.38 \\
Small-worldness & $2.138 \pm 0.676$ & $1.834 \pm 0.518$ & $1.965 \pm 0.485$ & 0.24 \\
\hline
\end{tabular}

This article is protected by copyright. All rights reserved. 


\begin{tabular}{lllll}
\hline GE & $0.704 \pm 0.070$ & $0.746 \pm 0.109$ & $0.695 \pm 0.105$ & 0.84 \\
Modularity (Q) & $0.620 \pm 0.026$ & $0.604 \pm 0.017$ & $0.604 \pm 0.026$ & 0.02
\end{tabular}

Data are presented as mean \pm standard deviation unless otherwise indicated. CCnorm $=$ normalized clustering coefficient; CPLnorm; normalized characteristic path length. 


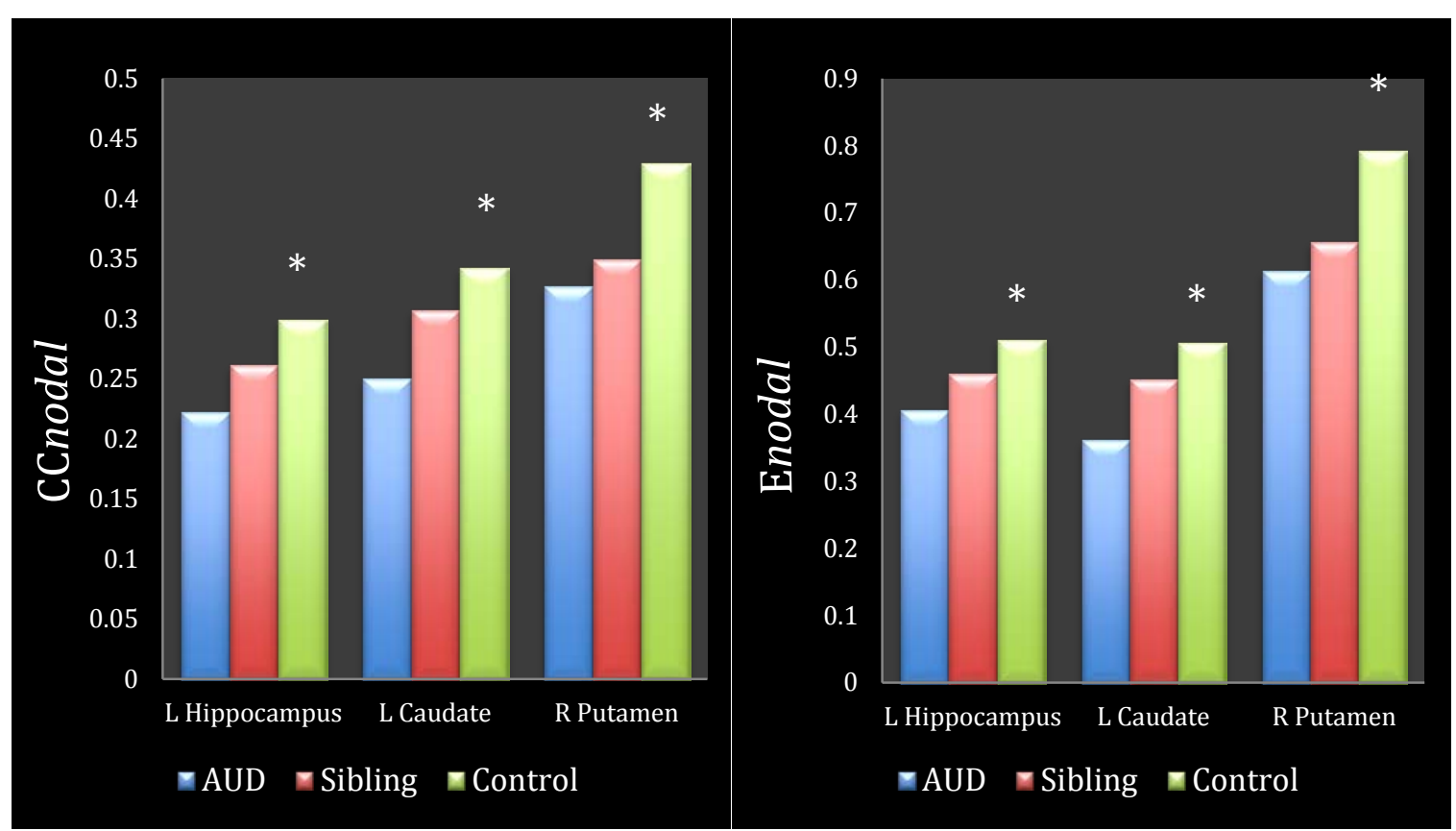

Figure 1 Nodes showed significant rank ordered differences, such that patients with alcohol use disorder (AUD) < Siblings < Controls for both nodal clustering coefficient (CCnodal) and nodal local efficiency (Enodal) after FDR correction.

* Post-hoc t tests demonstrated significantly reduced CCnodal and Enodal in AUD group compared with controls in all nodes. 


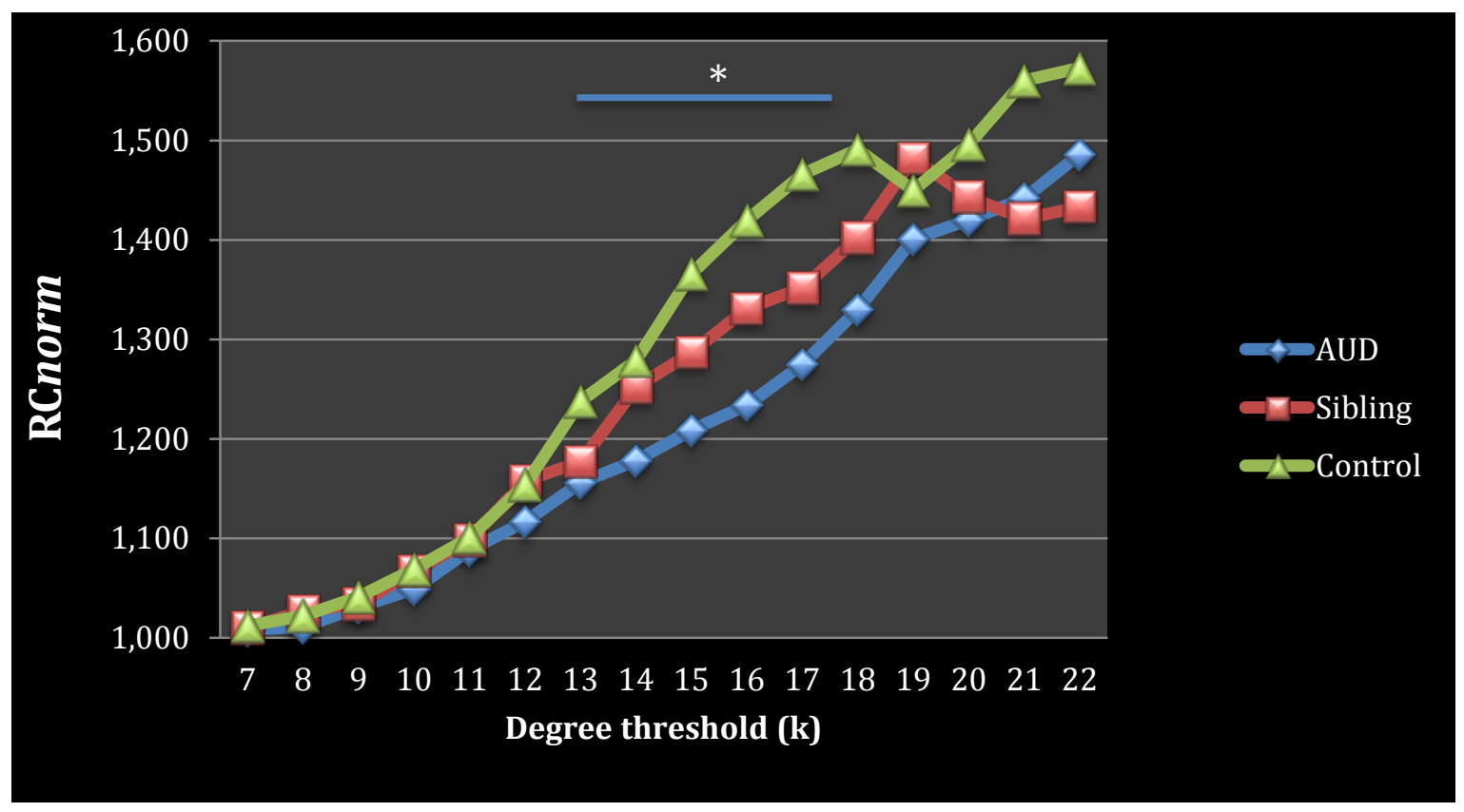

Figure 2 Groups showed rich club organization over a range of $\mathrm{k}$ from 7 to 22 .

* Normalized rich club coefficient (RCnorm) showed significant rank ordered differences, such that patients with alcohol use disorder (AUD) < Siblings < Controls over a range of k from 13 to 17 after FDR correction. Post-hoc t tests demonstrated significantly reduced RCnorm in AUD group compared with controls in all significant $\mathrm{k}$ levels. Siblings showed significantly reduced RCnorm in $\mathrm{k}=17(\mathrm{p}=0.046)$. 


\section{SFGdor.R}

\section{SMG.R}

SOG.R

IFGoperc.R

IFGtriang. $R$

Figure 3 Decreased structural connectivity in patients with alcohol use disorder compared to controls determined by network-based statistic analysis. R, right; SFGdor, superior frontal gyrus dorsolateral; IFGoperc, inferior frontal gyrus opercular part; IFGtriang, Inferior frontal gyrus triangular part; CAU, caudate; PUT, putamen; HIP, hippocampus; ITG, inferior temporal gyrus; SMG, supramarginal gyrus; SOG, superior occipital gyrus; MOG, middle occipital gyrus. 


\section{University Library}

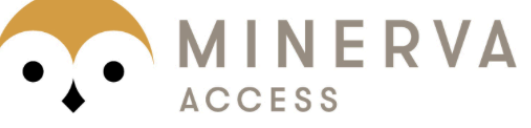

A gateway to Melbourne's research publications

Minerva Access is the Institutional Repository of The University of Melbourne

Author/s:

Zorlu, N;Capraz, N;Oztekin, E;Bagci, B;Di Biase, MA;Zalesky, A;Gelal, F;Bora, E;Durmaz, E;Besiroglu, L;Saricicek, A

Title:

Rich club and reward network connectivity as endophenotypes for alcohol dependence: a diffusion tensor imaging study

Date:

2019-03-01

Citation:

Zorlu, N., Capraz, N., Oztekin, E., Bagci, B., Di Biase, M. A., Zalesky, A., Gelal, F., Bora, E., Durmaz, E., Besiroglu, L. \& Saricicek, A. (2019). Rich club and reward network connectivity as endophenotypes for alcohol dependence: a diffusion tensor imaging study. ADDICTION BIOLOGY, 24 (2), pp.265-274. https://doi.org/10.1111/adb.12599.

Persistent Link:

http://hdl.handle.net/11343/294077 